\title{
SALINE INFUSION SONOGRAPHY IN ASSESSMENT OF ENDOMETRIAL PATHOLOGIES IN PATIENTS WITH THICKENED ENDOMETRIUM PRESENTING WITH AUB
}

\author{
Poornima Maravi1 ${ }^{1}$, Vijay Verma ${ }^{2}$, Lovely Kaushal ${ }^{3}$, Abhjeet Patil ${ }^{4}$, Chandraprakash Ahirwar $^{5}$ \\ ${ }_{1}^{1}$ Assistant Professor, Department of Radiodiagnosis, Gandhi Medical College, Bhopal. \\ ${ }^{2}$ Associate Professor and HOD, Department of Radiodiagnosis, Gandhi Medical College, Bhopal. \\ 3 Professor and HOD, Department of Radiodiagnosis, BMC, Sagar. \\ ${ }^{4}$ Associate Professor, Department of Radiodiagnosis, Gandhi Medical College, Bhopal. \\ ${ }^{5}$ Assistant Professor, Department of Radiodiagnosis, Gandhi Medical College, Bhopal.
}

\begin{abstract}
Abnormal Uterine Bleeding (AUB) is one of the commonest complaint of adult females presenting into the gynaecologic departments. In most of the patients the bleeding is due to the functional endometrial lesion, while the cause is hormonal in rest of the patients. These patients usually need invasive procedures like hysteroscopy or HSG for evaluation of intracavitary lesion. The aim of this study is to assess the role of Saline Infusion Sonography (SIS) in detecting the intracavitary (endometrial) lesions of uterus in patient with abnormal uterine bleeding and thickened endometrium on Transvaginal Ultrasound (TVS) and establishing its diagnostic accuracy.
\end{abstract}

\section{METHOD}

This prospective hospital-based study includes SIS examination of 80 adult female patients who presented with abnormal uterine bleeding and demonstrated thickened endometrium on transvaginal ultrasound at Department of Radiodiagnosis and Imaging, Gandhi Medical College and Hamidia Hospital, Bhopal (Madhya Pradesh), for the period of six months. Written informed consent from all the patients taken. All patients were evaluated on the same day and findings were recorded.

\section{RESULT}

60 patients out of 80 showed presence of functional endometrial lesion. Most common endometrial pathology was submucosal myoma followed by endometrial polyps. Most rare cases were of Uterine Synechiae $\{2$ in number $\}$. SIS yielded high sensitivity and specificity for intracavitary lesions and diagnostic accuracy for submucosal myoma and endometrial polyp was $100 \%$.

\section{CONCLUSION}

SIS proved to be highly sensitive and specific method to diagnose intracavitary lesion of uterus. The method is minimally invasive, well tolerated and easy to carry out under hospital settings with very less incidence of post-procedural complications (No major complication in this study). It can be used to replace hysteroscopy to diagnose various intracavitary (endometrial) lesion of uterus.

\section{KEYWORDS}

Saline Infusion Sonography (SIS), Thickened Endometrium, AUB, Endometrial Pathologies.

HOW TO CITE THIS ARTICLE: Maravi P, Verma V, Kaushal L, et al. Saline infusion sonography in assessment of endometrial pathologies in patients with thickened endometrium presenting with AUB. J. Evolution Med. Dent. Sci. 2016;5(41):2498-2502, DOI: $10.14260 /$ jemds/2016/583

\section{INTRODUCTION}

Abnormal Uterine Bleeding (AUB) is a common gynaecological complaint in women attending outpatient department. Abnormal vaginal bleeding is one of the most common presenting complaints in women of any age seeking gynaecologic health care. Two of the most frequently used diagnostic tests to investigate the cause of the bleeding are endometrial biopsy and transvaginal sonography. The most worrisome cause of abnormal bleeding is endometrial carcinoma, yet benign aetiologies are far more prevalent including fibroids, polyps and endometrial atrophy.

Financial or Other, Competing Interest: None.

Submission 09-01-2016, Peer Review 26-03-2016,

Acceptance 01-04-2016, Published 20-05-2016.

Corresponding Author:

Dr. Poornima Maravi,

Assistant Professor,

Department of Radiodiagnosis,

Gandhi Medical College,

Bhopal,

Madhya Pradesh.

E-mail: maravipoornima@gmail.com

DOI: $10.14260 /$ jemds/2016/583
Endometrial biopsy and transvaginal sonography have equal sensitivities for carcinoma, but sonography is far more effective in diagnosing benign disease.(1) The causes may vary from simple dysfunctional uterine bleeding without any organic cause to the endometrial cancer. The cases of AUB usually need thorough investigation to rule out organic causes, especially at perimenopausal and postmenopausal age when the risk of endometrial carcinoma is high.(2-3) Transvaginal Sonography (TVS) plays an important role as the initial modality for evaluation of AUB.(1-5), but its ability for screening the lesions within the endometrial cavity is limited.

The finding of a thickened central endometrial complex seen on TVS is often non-specific and may be caused by an endometrial polyp, submucosal fibroids, endometrial hyperplasia, carcinoma or cystic atrophy. Focal lesions are underdiagnosed at TVS, because of limitations of the doublelayer thickness evaluation.(3-5) Transvaginal ultrasound is used conventionally as initial investigation of patients with abnormal uterine bleeding, but saline infusion sonography is a better technique to reliably distinguish focal intracavitary lesion from diffuse endometrial lesions.(2) 
Transvaginal sonography with saline infusion in patients with AUB has been proved valuable to assess the organic cause for bleeding and is also easily acceptable. Nowadays, it is employed more commonly and may be proved to be the first line of investigation to assess these patients after hysteroscopy and biopsy. It is an accurate method for diagnosing endometrial abnormalities with high sensitivity and specificity rates. $(4)$

Saline Infusion Sonography (SIS) is an easy technique in which the endometrial cavity is distended with saline during transvaginal ultrasonic examination and it permits single layer evaluation of the endometrial lining and enables the sonologist to reliably distinguish focal from diffuse endometrial pathologic conditions. Several studies in recent literature have indicated that saline infusion sonography can differentiate intracavitary masses from more diffuse endometrial thickening.(6-11)

This study was performed to evaluate the role of SIS in detecting the endometrial lesion in women with AUB and thickened endometrium. Although, the use of fluid enhancement in abdominal ultrasound was described both for uterine and tubal observations.(10) it was not widely used until more recently.

\section{MATERIAL AND METHOD}

This hospital based, observational study was performed in Gandhi Medical College and Hamidia Hospital, Bhopal, Madhya Pradesh, for the period of six months starting from mid Feb 2015 study. All patients were referred from Gynaecological Department and were complaining AUB. A thorough and brief history was taken. Age ranged from 21-47 years. Patients were initially examined with transvaginal ultrasound and patients with thickened endometrium, focal and diffuse abnormality were further examined with saline infusion sonography. Phillips HD 7 colour Doppler scan with curved array vaginal transducer was used for all the examinations. Written and informed consent was taken from all the patients. All SIS examinations were performed on the same day.

Patient was placed in lithotomic position; cervix was visualized with speculum and a balloon catheter was inserted slowly. Keeping in place the bulb was inflated to make it tight at the external os and then $10 \mathrm{~mL}$ of sterile saline was slowly injected. At the same time vaginal transducer was inserted into the vagina and any focal or diffuse abnormality in uterus or endometrium was noted. The maximum thickness of endometrium was taken from one myo-endometrial interface to another myo-endometrial interface in longitudinal scans. Images were tagged and saved for further analysis.

Endometrium was regarded as abnormal if it was equal to or thicker than $8 \mathrm{~mm}$ in proliferative phase and equal and $>14$ $\mathrm{mm}$ in secretory phase in premenopausal women. Endometrium equal to or thicker than $5 \mathrm{~mm}$ in the postmenopausal period was regarded as abnormal.(10-11) In few patients, the technique was unsuccessful due to continuous leakage of saline through internal os. Few patients also complained of crampy lower abdominal pain, after which the procedure was temporarily stopped. Patients complaining heavy vaginal bleeding were excluded. None of the patients underwent/were on hormonal therapy or HRT during this study. Following table reference was taken to identify abnormally thickened endometrium.

\begin{tabular}{|c|c|}
\hline Menstrual Phase & Bilayer Thickness (MM) \\
\hline Proliferative phase & $4-8$ \\
\hline Secretory phase & $7-14$ \\
\hline Postmenopausal & $4-8$ \\
\hline \multicolumn{2}{|c|}{ Table 1: Bilayer Endometrial Thickness } \\
Pre- and Post-Menopausal Women
\end{tabular}

The saline by filling the endometrial cavity and providing dark background against the lesion helps clearly identify the abnormality, especially submucosal fibroid and endometrial polyps. Analysing the data after examinations, sensitivity, specificity, positive and negative predictive value of SIS for diagnosing various intracavitary lesions were noted. Results were compared to the final diagnosis made by histopathology.

\section{RESULTS}

All patients were adult females complaining of AUB, age ranging from 21-47 yrs. Overall, prevalence of endometrial lesion was $75 \%$ in adult females who presented with AUB and showing thickened endometrium. Most common lesion detected was submucosal myoma (31.66\%) followed by endometrial polyp (23.33\%); 3rd most common lesion was endometrial hyperplasia followed by cases of endometritis. Least common were cases of adhesions, while cases of endometrial carcinoma and retained products (RPOC's) share the equal incidences, though they were found in grossly different age groups. Submucosal myomas and endometrial polyps were commonly found in age group 25-45 yrs.; endometrial hyperplasia was common with age group 35-45. Endometrial carcinomas were found in older age group belonging to the patients $>45 \mathrm{yrs}$.

Total 60 patients were positive for various types of functional endometrial lesion out of 80 . True positives were 58 $(96.66 \%)$ patients and false positives were $2(3.33 \%)$ patients. True negatives were 18 (30\%) patients and false negatives were only $2(3.33 \%)$ patients.

Overall sensitivity and specificity of SIS for detecting endometrial lesions in patients with AUB and thickened endometrium was high measuring 0.96 and 0.90 respectively. The discrepancy between SIS and final diagnosis was present in 9 cases. The false positive diagnoses included $3 \mathrm{EH}, 2$ endometrial carcinoma, 4 false negative diagnoses were made in 9 cases which included 3 endometritis and 1 case of uterine synechiae. Three false negative results included chronic endometritis and case of endometrial polyp, uterine synechiae each.

The tolerance of the saline infusion sonography was excellent. In a few patients, some endovaginal reflux of saline was noted during injection; 8 patients complained of pelvic discomfort and during examination and injection was stopped temporarily in 2 patients because of the patient's complaint of crampy pelvic pain.

\section{DISCUSSION}

AUB has been one of the most common complaints in between the women attending gynaecologic OPDs. Vast number of these women is pre- or peri-menopausal. Though in many cases the bleeding is purely hormonal, major cases also demonstrates single or combined endometrial pathology as a cause of bleeding and needs to be evaluated. Transvaginal ultrasound has remained basic tool to investigate the patients with AUB. 
Extensive work has been done evaluating its diagnostic ability to evaluate intracavitary and endometrial lesion. Though it has been vastly used for last few decades, there is more concentration to establish SIS as a basic tool to investigate these patients. Despite the widespread use of TVS for initial evaluation of AUB, the number of studies in the literature is growing which indicate that TVS alone has its limitations in depicting small nodular lesions, which are isoechoic within the endometrium and even a normal thickness endometrium may be seen to represent endometrial hyperplasia.(12-14) Sensitivity being 87\% (Range 24-96\%) and the specificity $82 \%$ (range $29-93 \%) .(10,12)$

Similarly, TVS cannot reliably differentiate submucosal from intramural leiomyoma in many instances, which is an important distinction for hysteroscopic resection of these lesions.(6) Our study results indicate that all 19 cases of submucous myomas were correctly diagnosed and located by SIS. These results also simulate with results by Laifer-Narin et al(13) who claim that $14 \%$ of 114 patients showing normal TVS findings revealed abnormalities on SIS. There are many advantages of using saline infusion sonography as an initial evaluation test in abnormal uterine bleeding.

Literature identified the major functional cause with AUB in these patients are submucosal fibroids and endometrial pathologies, which interfere with normal functioning endometrium and leads to irregular bleeding. Because SIS by distending the uterine cavity with saline/fluid and making a clear interface between the lesion and dark endometrial cavity reliably distinguish submucosal myoma from intramural myomas. Similarly, endometrial polyps which are seen suspended in cavity can be easily made out. The sensitivity and specificity of SIS have been reported to be as high as $85-91 \%$ and $83-100 \%$, respectively. $(3,9,11,14)$ Our result have shown 0.96\% Sensitivity, 0.90\% Specificity, 0.96\% PPV, and 0.90\% NPV for SIS to diagnose intracavitary/endometrial lesions. Similar high accuracy rates ranging from $84 \%$ to $96 \%$ have been reported by other studies. $(7,11)$

In our study three most commonly seen lesions were submucous myoma, endometrial polyps and hyperplasia. There have been several reports of the diagnostic value of SIS in differentiation of these diseases. Epstein et al(7) has reported an almost perfect agreement (96\%) between SIS and hysteroscopy in the diagnosis of focally growing lesions. SIS and hysteroscopy both had a sensitivity of approximately $80 \%$ with regard to diagnosing endometrial polyps (false-positive rates of $24 \%$ and $6 \%$ respectively), whereas conventional ultrasound can miss half of the polyps (sensitivity, $49 \%$; falsepositive rate, $19 \%$ ). M Aslam et al(2) in a study of 106 patients with AUB has achieved $93.3 \%$ sensitivity, $94.6 \%$ positive predictive value and $93.3 \%$ diagnostic accuracy in the detection of endometrial polyps by SIS.

In the present study 5 cases of endometrial carcinoma were detected, 2 out of 5 were misdiagnosed as endometrial hyperplasia on SIS; this may be because both of the cases containing very small lesion associated with early atypia detected on endometrial biopsy. Epstein et al(7) has reported that hysteroscopy is superior to TVS and SIS discriminating between benign and malignant lesions (sensitivity $84 \%, 44 \%$ and $60 \%$; false-positive rate $15 \%, 6 \%$ and $10 \%$, respectively). However, biopsy is always needed to differentiate between benign and malignant endometrial pathologies.
Dubinsky et al(1) established criteria for classifying benign and suspicious lesions seen on SIS. Their results for detecting endometrial carcinoma with SIS had a sensitivity of $89 \%$, specificity of $46 \%$, PPV of $16 \%$ and NPV of $97 \%$. These criteria were highly predictive for benign processes, particularly endometrial thickening. However, because many pathologic conditions had a suspicious appearance, the positive predictive value of SIS for carcinoma may be low. Most endometrial abnormalities including early carcinoma, appear as a focal endometrial thickening/mass on transvaginal Ultrasonography; therefore, women with all endometrial functional lesions should undergo biopsy and histopathological correlation.

8 cases of procedural failures were found in this study, which was due to failure of compliance of patients and leakage of saline through internal os. No specific uterocervical lesion can be demonstrated in these patients, which may lead to the procedural failure. Failure rate of $2.9 \%$ to $6 \%$ is mentioned in the literature, which is mainly due to cervical stenosis, commonly present in postmenopausal patients. Majority of our study population (76.66\%) were premenopausal, which was consistent with most of previous studies.

Few complications of SIS mentioned in literature are vasovagal syncope, endometrial shearing with catheter, bleeding, infection, perforation (due to instrumentation) and theoretical risk of spreading of carcinoma. Dubinsky et al(1) found two cases of endometritis after SIS in their study ( $n=89$ patients). The risk of infection following SIS is estimated to be $1 \%$.(6) However, in our study all patients were well and did not complain of any sign of infection. Two patients complained crampy pelvic pain after the initiation of procedure; however, they tolerated the procedure well after temporary stoppage of procedure. This may be due to rapid distension of uterine cavity by the saline. No major complications found.

One of the limitations of our study is that we did not evaluate the patients with early miscarriages who were later diagnosed as containing RPOC's.

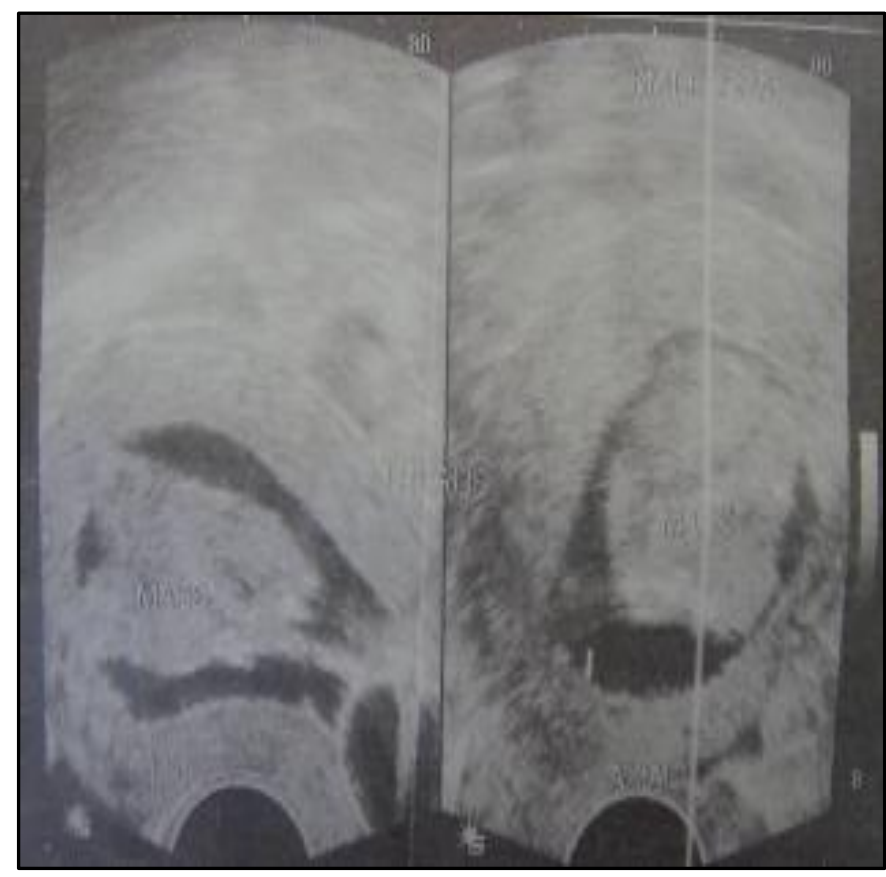

Fig. $A, B$ 


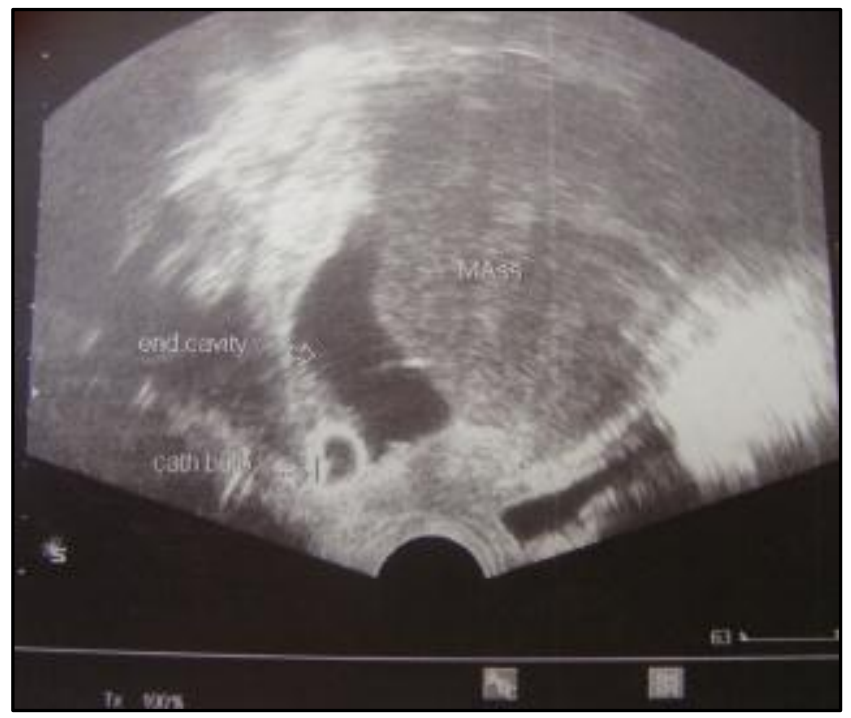

Fig. $C$

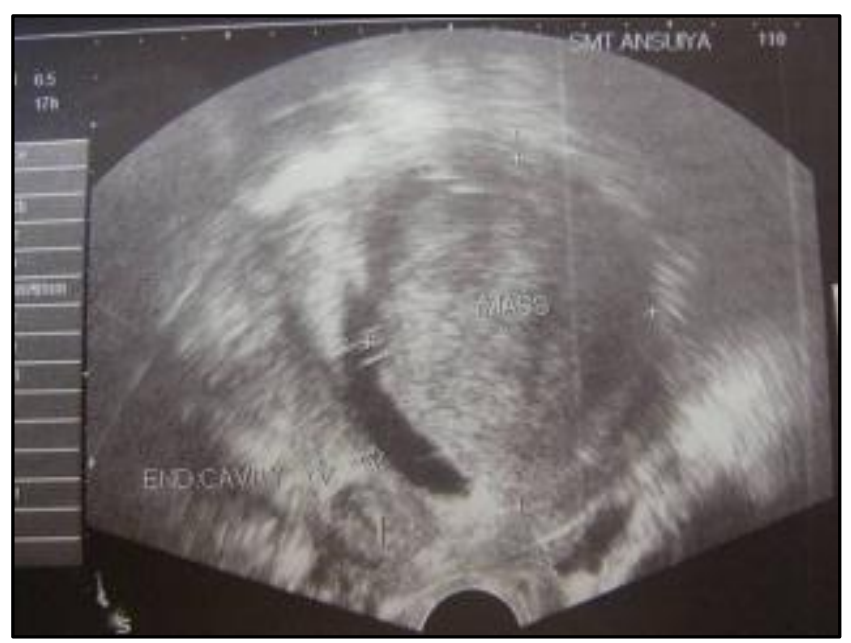

Fig. $D$

Figure a, b, c, d - showing large intracavitary masses which can be easily made out over the dark background of saline.

All 19 and 14 cases of submucosal myoma and endometrial polyps were correctly diagnosed and were consistent with the findings on hysteroscopically taken biopsy and histopathology. The sensitivity and specificity of SIS for diagnosing these lesions was high reaching to almost $100 \%$. Out of 9 cases of endometrial hyperplasia, 6 were correctly diagnosed on SIS.

Diagnosis and treatment of irregular uterine bleeding in perimenopausal women as well as postmenopausal women often warrants for a great deal of medical care. Treatment options also vary per patient; some patients with submucous myomas are inappropriate candidates for resectoscopic surgery, whereas in others it would be the treatment of choice. However, the major part of treatment for endometrial polyps was hysteroscopic removal, until and unless they are multiple or found to be malignant. Treatment for endometrial hyperplasia is early hysterectomy and hormonal therapy if needed.

In this prospective hospital based study, saline infusion sonohysterography was performed in 80 women who had unexplained perimenopausal uterine bleeding with thickened endometrium; 14 cases of polypoid lesions were found, all underwent operative hysteroscopic removal except 2 cases of multiple polyps who were kept under the expectant management.

All 12 cases of endometrial polyps were found to be benign on histopathology. Submucosal myomas were found in 19 patients, 8 of which were treated with hysteroscopy removal, 6 undergone total hysterectomy and rest were kept on expectant management.

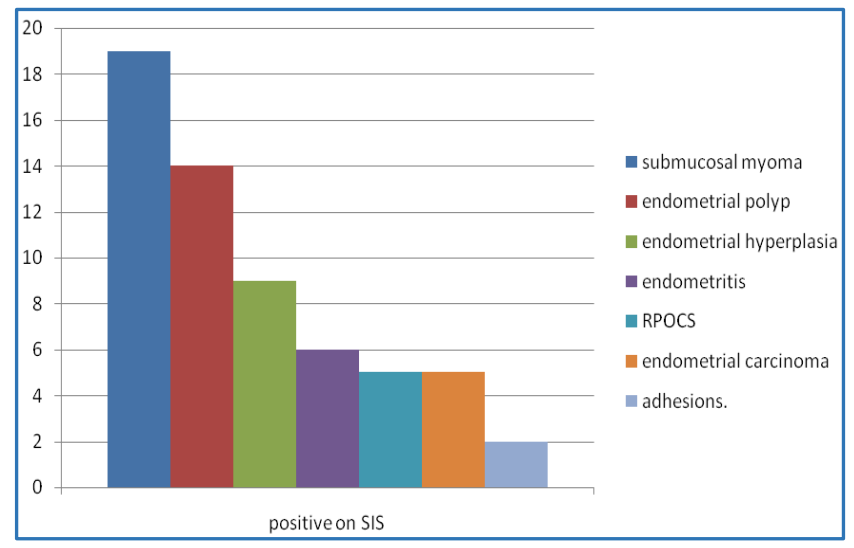

Fig. 1: Showing Incidence of Endometrial Lesions found on SIS

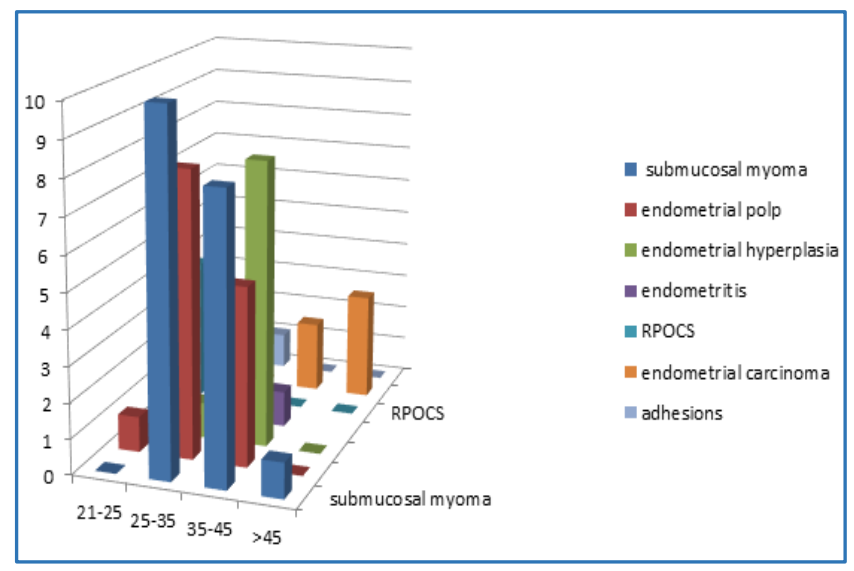

Fig. 2: Age Wise Distribution of Endometrial Lesions on SIS

\begin{tabular}{|c|c|c|c|}
\hline & $\begin{array}{c}\text { Estimated } \\
\text { Value }\end{array}$ & \multicolumn{2}{|c|}{$\begin{array}{c}\text { 95\% Confidence } \\
\text { Interval }\end{array}$} \\
\hline & & $\begin{array}{c}\text { Lower } \\
\text { Limit }\end{array}$ & $\begin{array}{c}\text { Upper } \\
\text { Limit }\end{array}$ \\
\hline Prevalence & 0.75 & 0.63847 & 0.837158 \\
\hline Sensitivity & 0.966667 & 0.874512 & 0.999205 \\
\hline Specificity & 0.90 & 0.668723 & 0.982487 \\
\hline PPV & 0.966667 & 0.874512 & 0.994205 \\
\hline NPV & 0.90 & 0.668723 & 0.982487 \\
\hline \multicolumn{2}{|c|}{ Table 2: Showing Sensitivity, Specificity, PPV and } \\
NPV of SIS for Different Endometrial Lesion \\
\hline
\end{tabular}

- $\quad$ PPV- Positive Predictive Value.

- NPV- Negative Predictive Value. 


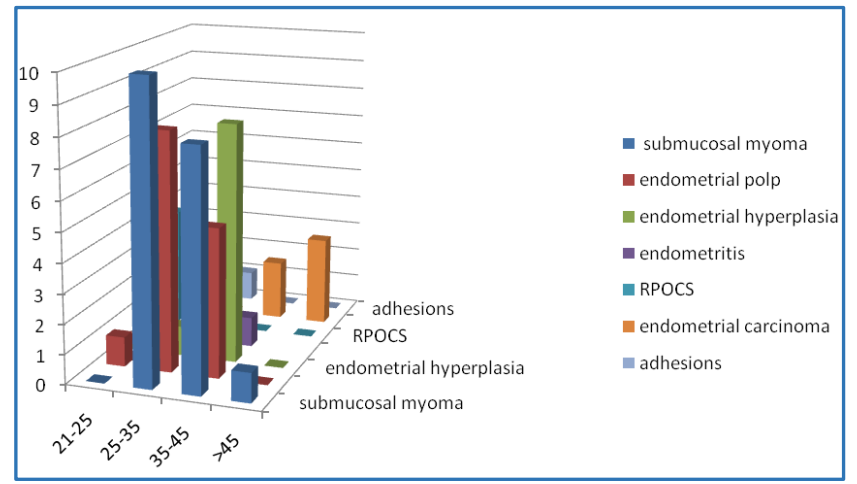

Fig. 3: Comparison of Diagnostic Performance of SIS with HP

- $\quad$ SIS-Saline Infusion Sonography.

- HP-Histopathology.

\section{CONCLUSION}

Saline infusion sonography to enhance visualisation of intracavitary lesion of uterus in pre/postmenopausal women could reliably distinguish patients with functional intracavitary (Endometrial) lesion. Furthermore, it was found that polyps can be distinguished from submucous myomas with high degree of confidence. The need for diagnostic hysteroscopy in patients whose bleeding is dysfunctional is therefore eliminated. When changes are focal as is the case in polyps, some hyperplasias and some carcinomas, they can be appreciated as such with fluid-instillation sonohysterography, and directed biopsies can be carried out. Saline infusion sonography can be used as alternative to hysteroscopy for investigation of AUB and thickened endometrium at hospital settings with high yielding results.

\section{REFERENCES}

1. Dubinsky TJ. Value of sonography in the diagnosis of abnormal vaginal bleeding. J Clin Ultrasound 2004;32(7):348-53.

www.ncbi.nlm.nih.gov/pubmed/1529330

2. Muhammad Aslam, Lubna Ijaz, Shamsa Tariq, et al. Comparison of transvaginal sonography and saline contrast sonohysterography in women with abnormal uterine bleeding: correlation with hysteroscopy and histopathology. Int J Health Sci (Qassim) 2007;1(1): 17-24.

3. O'Connell LP, Fries MH, Zeringue E, et al. Triage of abnormal postmenopausal bleeding: a comparison of endometrial biopsy and transvaginal sonohysterography versus fractional curettage with hysteroscopy. Am J Obstet Gynaecol 1998;178(5):956-61.
4. Abu-Ghazzeh Y, Shakoury WA, Barqawi R. Comparative study of transvaginal hysterosonography and biopsy for the evaluation of post-menopausal bleeding. Ann Saudi Med 1999;19(2):116-9.

5. Davis PC, O'Neill MJ, Yoder IC, et al. Sonohysterographic findings of endometrial and subendometrial conditions. Radiographics 2002;22(4):803-16.

6. Dueholm M, Forman A, Jensen ML, et al. Transvaginal sonography combined with saline contrast sonohysterogram in evaluating the uterine cavity in premenopausal patients with abnormal uterine bleeding. Ultrasound Obstet Gynaecol 2001;18(1):5461.

7. Epstein E, Ramirez A, Skoog L, et al. Transvaginal sonography, saline contrast sonohysterography and hysteroscopy for the investigation of women with postmenopausal bleeding and endometrium $>5 \mathrm{~mm}$. Ultrasound in Obstet Gynaecol 2001;18(2):157-62.

8. Farquhar C, Ekeroma A, Furness S, et al. A systematic review of transvaginal ultrasonography, sonohysterography and hysteroscopy for investigation of abnormal uterine bleeding in premenopausal women. Acta Obstet Gynaecol Scand 2003;82(6):493-504.

9. Ryu JA, Kim B, Lee J, et al. Comparison of transvaginal ultrasonography with hysterosonography as a screening method in patients with abnormal uterine bleeding. Korean J Radiol 2004;5(1):39-46.

10. Randolph JF, Ying YK, Maier DB, et al. Comparison of realtime ultrasonography, hysterosalpingography, and laparoscopy/hysteroscopy in evaluation of uterine abnormalities and tubal patency. Fertil Steril 1986;46(5):828-32.

11. Getpook C, Wattanakumtornkul S. Endometrial thickness screening in premenopausal women with abnormal uterine bleeding. J Obstet Gynaecol Res 2006;32(6):58892.

12. Nasri MN, Coast GJ. Correlation of ultrasound findings and endometrial histopathology in postmenopausal women. Br Med J 1989;96(11):1333-8.

13. Lewit N, Thaler I, Rottem S. The uterus: a new look with transvaginal sonography. J Clin Ultrasound 1990;18(4):331-6.

14. Milingos S, Kallipolitis G, Stefanidis $\mathrm{K}$, et al. Saline contrast hysterosonography in infertile patients and in women with abnormal uterine bleeding. Eur J Gynaecol Oncol 2005;26(5):564-70. 